\title{
NOTHING IS CLEAR AS IT SEEMS A TRICKY CASE OF STROKE
}

Poretto V. ${ }^{1}$, Piffer S. ${ }^{1}$, Motta A. ${ }^{2}$, Bonvini S. ${ }^{3}$, D’Onghia G ${ }^{4}$, Bommassari R. ${ }^{4}$, Graffigna A $^{2}$, Giometto B. ${ }^{1}$

1 Department of Neurological Disorders, Neurology, Santa Chiara Hospital, Trento, Italy; 2 Department of Cardiovascular Diseases, Cardiosurgery, Santa Chiara Hospital, Italy; 3 Department of Cardiovascular Disease, Vascular Surgery, Santa Chiara Hospital,

Trento, Italy; 4 Department of Cardiovascular Disease, Cardiology, Santa Chiara Hospital, Trento, Italy

Background. To date, several studies has focused on stroke etiology, given its great importance in correctly setting the secondary prevention and to avoid recurrences. Nevertheless, a relevant percentage of strokes still remains cryptogenic. In order to better stratify cryptogenic strokes, a neuroradiological classification has been proposed based on infarct morphology and topography, introducing also the concept of embolic stroke of undetermined sources (ESUS).

It is however important to bear in mind that the diagnostic reliability of such a classification may not reflect the actual clinical situation of the patient.

Case report. A 64-years old woman referred to our hospital for fuzzy vision and dizziness, with evidence of bilateral upward gaze palsy and no convergence on neurological examination. She reported a history of smoking-habit, hypertension, dyslipidaemia and autoimmune setting with tiroiditis, rheumatoid arthritis and systemic scleroderma.

\section{Investigations}

- acute CT scan excluded hemorrhages and an AngioCT did not reveal relevant extra and intracranial stenosis

- no alteration in cardiac rhythm detected on the ECG-monitoring

- MRI evidence of a minute $(0.5 \mathrm{~mm})$ recent thalamo-mesecephalic infarct

(Figure 1), suggestive for lacunar etiology.

She was therefore put on an antiplatelet treatment.

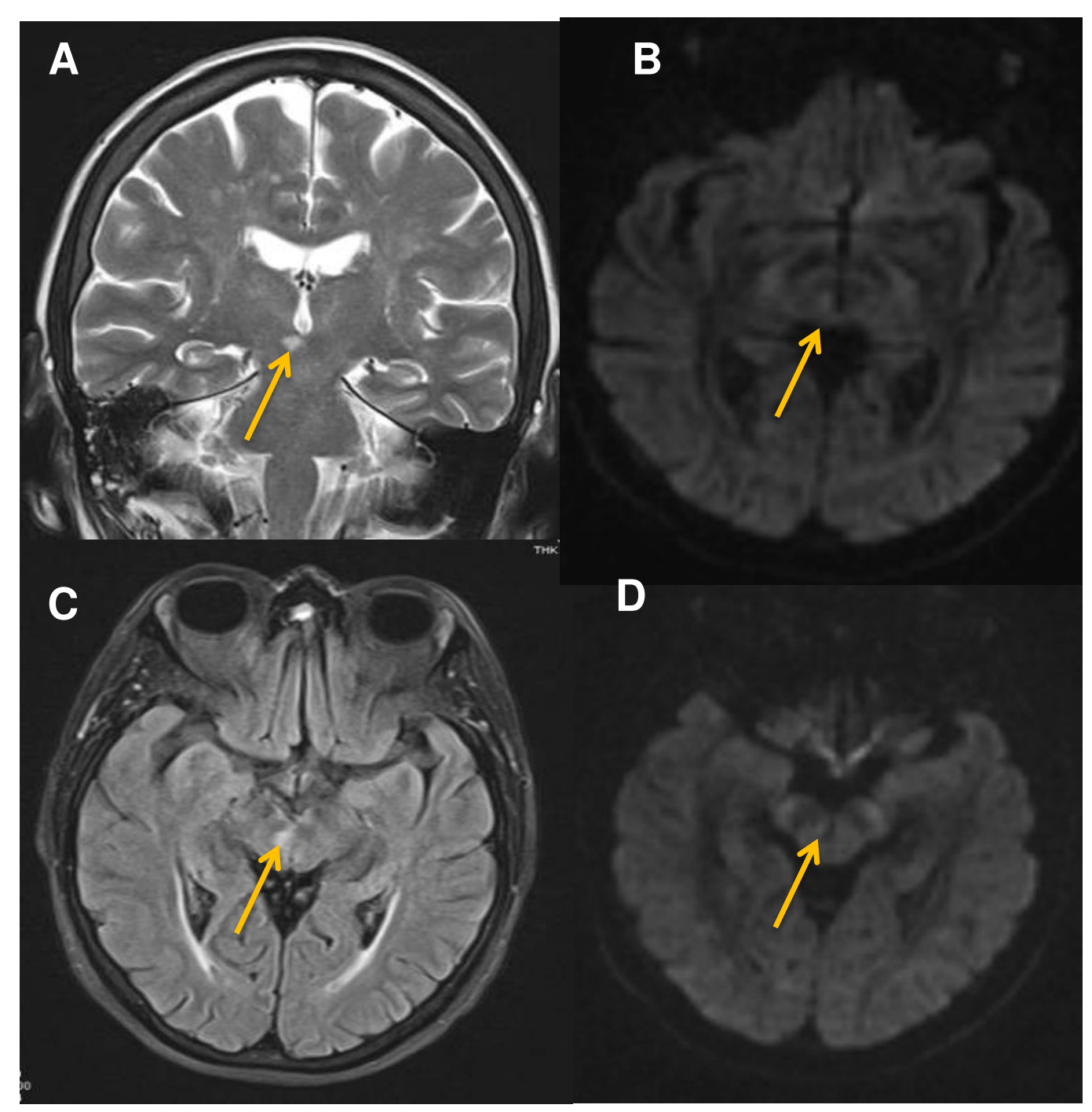

Figure 1. MRI images of patient's infarct.

Panel A reports a coronal T2-weighted image showing a minute lesion at thalamic-mesecephalic junction, that is evident on FLAIR image (C) and shows diffusion-restriction on two axial consecutive DWI slices (B and D)

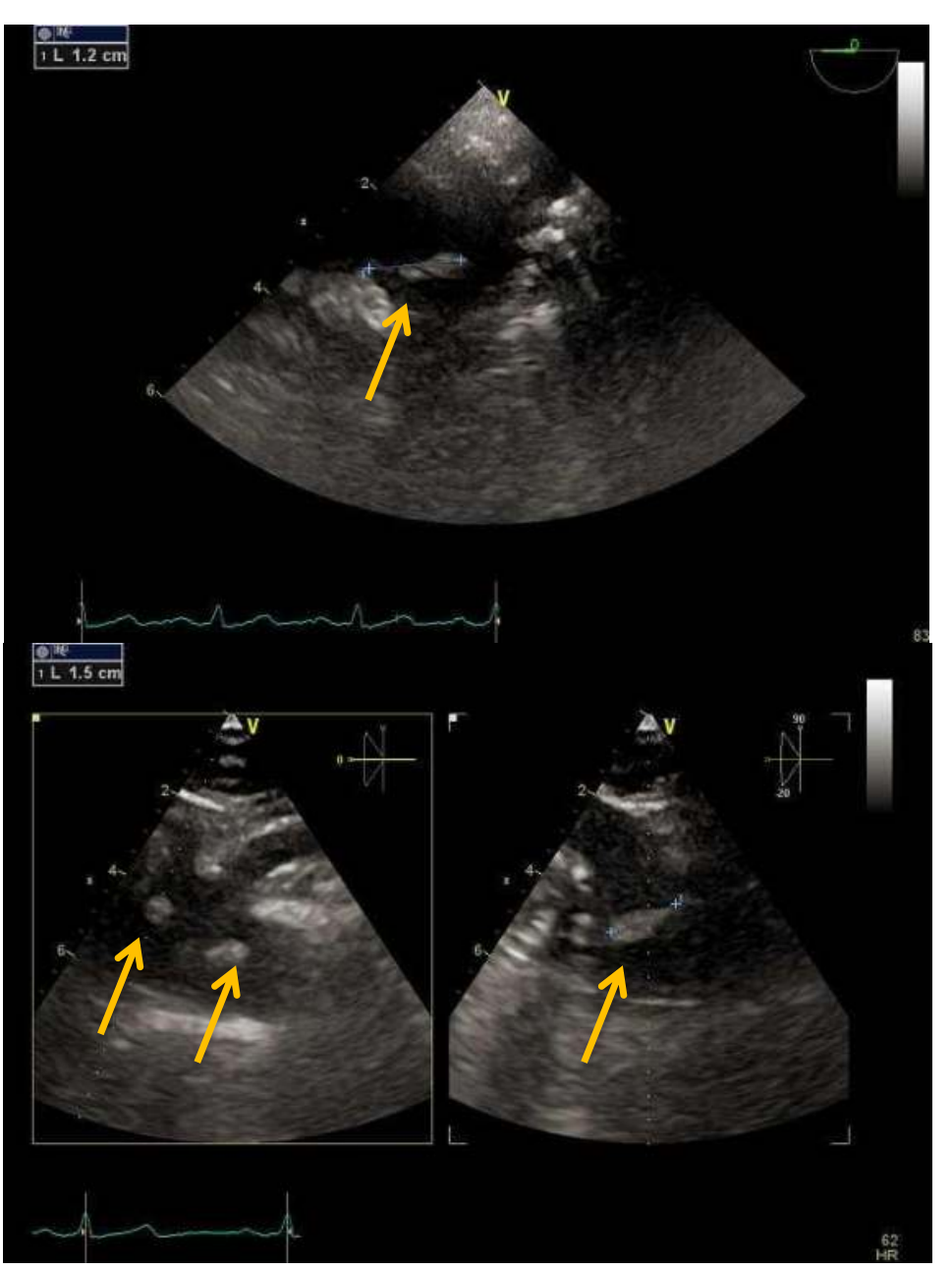

Figure 2. Echocardiografic images. Both panels show the presence of diffuse aortic arch atherosclerosis two pedunculated thrombi, protruding into brachiocefalic artery and left common carotid artery
- A transthoracic echocardiogram showed extensive atherosclerosis of aortic arch (Figure 2), which was further investigated through a trasesophageal echo, revealing two pedunculated and mobile thrombi protruding into brachiocephalic artery.

\section{Treatment choice}

Anticoagulation with LMWE was then promptly started.

However, during the following day of hospital stay, patient experienced acute arm ischemia of the right radial artery and was thus treated with embolectomy.

Patient was ultimately referred to a cardiosurgical for substitution of the aortic arch, with surgical success.

Conclusions. Despite anamnesis and infarct MRI-findings were misleading and suggestive of a lacunar etiology, a comprehensive diagnostic workup allowed the identification a microembolization from aortic plaques as the cause of the reported symptoms in a patient.

This enabled to correctly classify the observed lesion as embolic, with remarkable consequences on the therapeutic strategy. 